\section{The effect of structure on scanning strategies}

\author{
E. C. T. WALKER
}

Massachusetts Institute of Technology, Cambridge, Massachusetts 02139

Ss heard four-digit strings and saw a two-digit probe immediately after each. Every possible combination of two digits taken from the string, as well as each member of the string combined with some digit from the negative set was tested. Reaction times for verifying that both probe digits occurred in the string reveal that Ss grouped the members of the string into two ordered pairs. This result argues that the structure Ss might assign to so-called random strings must be considered when discussing scanning strategies. Structural explanations of two common patterns of reaction times are presented.

When Sternberg (1969b) asked Ss to recognize whether two digits had appeared in the same or reverse order in a string, their reaction times (RT) increased with the serial position of the digits in the string. The slope of the increase in RT was the same for probes containing digits in either the same or reversed orders. Sternberg argued that this pattern of RT suggested that the test digits were located with a serial, self-terminating scan.

His experiment included strings that were $3,4,5$, and 6 digits long. And, indeed, the increase in RT with serial position was quite regular when strings of all lengths were considered (Fig. 1).

However, RT to probes of strings containing an even number of digits apparently differed from those of strings with an odd number: in strings four and six digits long, probes containing the last two digits were recognized faster than those containing the preceding two digits, and the same was true of probes

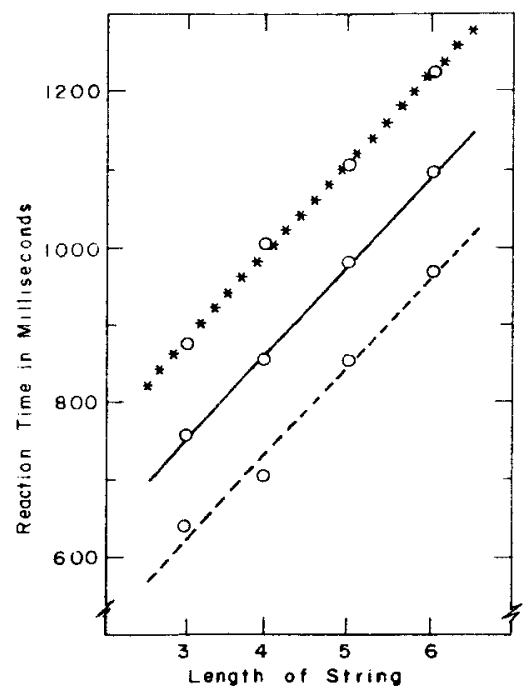

Fig. 1. Latencies of correct same (lower) and different (upper) responses and their mean (center). From Sternberg (1969b). containing the middle two digits of six-digit strings (Fig. 2). In other words, RT for successive probes of strings with an even number of members seems not to increase regularly.

Such strings form well-defined sequences of pairs, 12-34-56- etc., while those strings with an odd number of members must have one member excluded from an arbitrary pairing. This coincidence suggests that not only the serial order of the digits, but also the structure of the string-in this case, grouping into pairs-affects recognition latencies.

Of course, the apparent differences found in Fig. 2 might have arisen from some gross property of recognizing members of such strings, rather than from structure. Suppose, for example, that the probe representing Positions 3 and 4 in a string of four digits is recognized more quickly than other probes. Such a result might be attributed either to the probe's containing the last digit of the string or to its containing digits that were members of the same pair in the string.

If the first explanation were correct, other probes containing the last digit also should be recognized relatively explanation were correct, probes containing paired digits should be recognized relatively quickly, no matter which serial positions those digits represented. Provided that explanations based on the serial positions of individual digits failed, the latter result would be evidence that the structural organization of such strings affects recognition latency.

To test the idea that Ss do assign structure to such digit strings, probes containing every set of two digits taken from a string four digits long were tested. If Ss assign the structure shown at the top in Fig. 3 , then probes that represent pairs of digits should be recognized more quickly than those representing digits that are not pairs.

In order that the same overt response be made to probes with both same and different orders, "catch" probes that contained one digit from quickly; while if the second the string and one digit from the negative set were used. Assuming that all digits from the negative set are recognized with the same latency, latencies for recognizing individual digits from the positive set should appear in the results for catch trials. Consequently, RT from these catch probes provides a basis for comparing the recognition of individual digits with that of two digits.

\section{METHOD}

Ss were 24 undergraduate psychology students. Each $\mathbf{S}$ heard 136 strings of four digits in an uninterrupted experimental session. After each string, a two-digit probe was presented visually. Each $\mathbf{S}$ saw probes consisting of the 12 possible combinations of two digits from the string and of the 8 possible combinations of a member of the string with some digit from the negative set. For a typical string, 1234, the probes would be $12,23,34,24$, $13,14,1 x, 2 x, 3 x, 4 x$, and their reverses. Of all the trials, 64 contained negative-set digits. Consequently, each type of catch probe received eight trials, while each of the 12 probe types containing digits that both occurred in the string received six trials. The strings were composed by a computer routine, which permitted no digit to occur twice in a string or probe and assured that all the digits occurred equally often in each $S$ 's set of trials. The order in which the various probe types occurred was random.

$\mathrm{E}$ pressed a key to present the probe immediately after he had read the stimulus string aloud. The delay with which the probes were presented thus depended on E's reaction time to the end of the string. A timer started simultaneously with the presentation of the probe and stopped when $S$

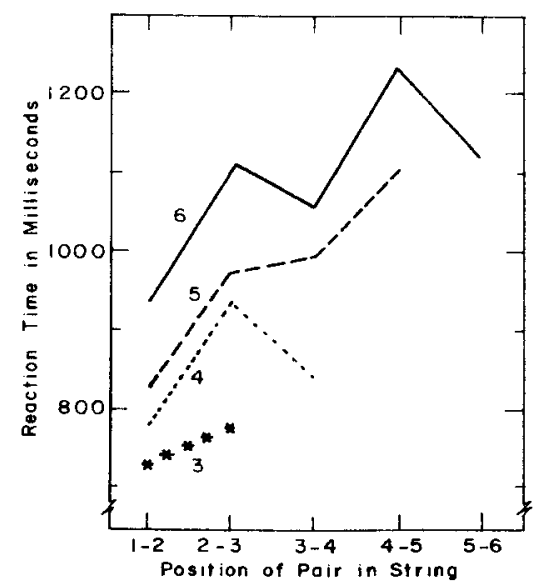

Fig. 2. Mean latencies of correct responses for strings of four lengths. From Sternberg (1969b). 


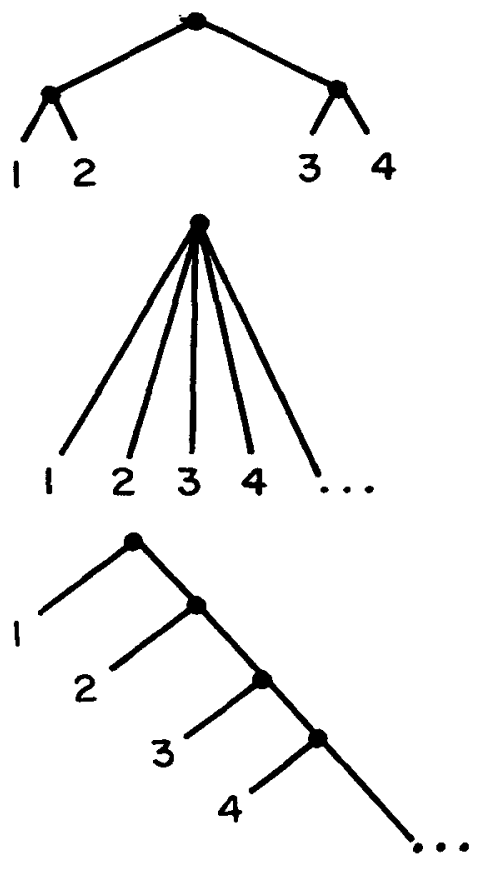

Fig. 3. Tree diagrams representing ( $t o p$ to bottom) pairs, multiple-branching, and right-branching structures.

pressed one of two keys to indicate whether both digits that he saw were in the string that he had just heard. E recorded response latency, changed stimulus cards, reset the apparatus, and proceeded with the next trial. Although simple instructions and an example were presented, no other training was given.

\section{RESULTS}

Analysis of variance revealed that probes that contained digits in the same order as they had occurred in the string were verified faster than those with different orders $(F=30.9$, $\mathrm{p}<.001$, df $=1 / 23)$. Probes containing digits that were contiguous in the string were recognized faster than those with separated digits $(F=$ 37.7, $\mathrm{p}<.001$, df $=1 / 23$ ). Catch probes in which the digit from the negative set occurred first were rejected faster than those in which it occurred second $(F=5.7, p<.025, \mathrm{df}$ $=1 / 23)$. No other effects or

Table 1

Response Latencies for Catch Trials (Seconds)

\begin{tabular}{lcccc}
$\begin{array}{c}\text { Position of } \\
\text { Negative } \\
\begin{array}{c}\text { Digit in } \\
\text { Probe }\end{array}\end{array}$ & \multicolumn{3}{c}{$\begin{array}{c}\text { Position of Positive } \\
\text { Digit in String }\end{array}$} \\
\cline { 2 - 5 } & 1 & 2 & 3 & 4 \\
\hline First & 1.09 & 1.10 & 1.11 & 1.10 \\
Second & 1.11 & 1.15 & 1.13 & 1.10 \\
\hline
\end{tabular}

Table 2

Response Latencies for Positive Conditions (Seconds)

\begin{tabular}{|c|c|c|c|c|c|c|}
\hline \multirow{2}{*}{$\begin{array}{l}\text { Order } \\
\text { of Digits } \\
\text { in Probe } \\
\text { and String }\end{array}$} & \multicolumn{2}{|c|}{ Position } & of Probe & Digits in & String & \\
\hline & 1,2 & 1,3 & 1,4 & 2,3 & 2,4 & 3,4 \\
\hline $\begin{array}{l}\text { Same } \\
\text { Different }\end{array}$ & $\begin{array}{r}.99 \\
1.11\end{array}$ & $\begin{array}{l}1.12 \\
1.18\end{array}$ & $\begin{array}{l}1.12 \\
1.22\end{array}$ & $\begin{array}{l}1.06 \\
1.13\end{array}$ & $\begin{array}{l}1.12 \\
1.22\end{array}$ & $\begin{array}{r}.98 \\
1.10\end{array}$ \\
\hline
\end{tabular}

interactions produced significant differences.

Consider first the results from the catch probes (Table 1). Since the probes in which the digit from the negative set occurred first are rejected more quickly, we can assume that the first digit of a probe is recognized first. If so, then reaction time (RT) for probes in which the digit from the negative set is recognized last should reveal the latency with which the first digit that actually occurred in the string is recognized. RT for these probes did not differ by position. Therefore, variations in RT for probes containing digits that both occurred in the string (positive condition) are likely to have arisen either from recognizing the second digit or from recognizing two digits together. $\mathrm{RT}$ for probes containing the same second digit varied widely (Table 2 ), so the former suggestion must be rejected. It seems, instead, that the pattern of RT reflects some organization of the string. That is to say, the results appear to arise not from the successive or simultaneous recognition of individual digits taken from an unstructured positive memory set, but rather from the recognition of two digits taken as a unit that is part of a structured string.

The most obvious relation among the digits in a string is their serial order. One might propose that $S s$ simply scanned serially to locate the first digit of a probe and proceeded to the second digit, or, in the case of reverse order probes, returned to the beginning of the string to rescan and locate the second digit. In that case, contiguous digits would be recognized more quickly than separated digits, as was the case. However, there are six instances of contiguous digits in a string with four members; the first two digits, the last two digits, the middle two digits, and their reverses. If contiguity were the sole determinant of RT, RT for probes of the middle two digits should not differ from that for the first and last two, but it did ( $p<.05$, Newman-Keuls test). Similarly, as the separation of two digits increased, e.g., probes of the first and third digits compared to those of the first and fourth, RT should increase; yet, it did not (p > 05, Newman-Keuls test). Certainly, one might counter such evidence with the obvious suggestion that probes containing digits from first and last positions are recognized more quickly. However, the results for the catch trials contained no such effect.

More compelling evidence against such a scanning process is contained in the results for reverse order probes. If the string were simply rescanned serially to find the second probe digit, those probes containing contiguous (but reversed) digits would have large RT; yet, they were recognized most quickly of the reverse order probes.

Similar inconsistencies await other attempts to explain the pattern of RT by referring only to the scanning processes that Ss might employ. Consider, instead, that these strings, which have no obvious structure other than the serial order of their members, are organized as two ordered pairs by Ss. Then probe digits that represent a pair from the string should be recognized more rapidly than digits that are not pairs, and digits that are not pairs should be equally recognizable, among themselves. That the structure assigned includes order is reflected by the consistently larger $R T$ that a disagreement of order in the string and probe produces.

Suppose that the scanning process must begin at the top node in a structured string like that shown at the top of Fig. 3. Since paths to individual digits are equivalent, the catch probes should show no direct evidence of structure. However, probes in the IN condition should reveal the effects of structure. In particular, those probes containing digits that represent a pair should be recognized more rapidly than those containing digits that break this pairing. Of course, strings only four digits long are too short to provide evidence about the sequence in which the pairs themselves are scanned, but the evidence that scanning to find a digit from each pair requires more time than scanning a single pair is clear.

The results of the experiment reported here display in microcosm the paradox into which trying to account for RT without referring to structure leads. In the catch trials, RT appears not to be influenced by serial position. Yet the RT for the probes with occurring digits-considering them as reflections of serial position rather than structure-are 
systematically correlated with serial position. To account for RT by referring to a scanning procedure alone, one would have to posit a scan which, on the one hand, reflected serial position and, on the other, did not. Incidentally, yet a third procedure would be required for dealing with reverse order probes. When the possibility that Ss assign structure to the input strings is introduced, the paradox disappears.

Clearly, the structural organization of the digits in the string strongly affected recognition latencies. Whatever the scanning process, it interacted with structural variables to produce the experimental results. That being the case, attempts to discover the manifestations of scanning procedures in patterns of $R T$ must consider what structure Ss might assign to strings in order to accomplish the tasks imposed by the experiment.

The structural hypothesis may be extended to suggest that in experiments which require recognizing single items from such strings, Ss assign one of two structures. When the retention of order information is not required by the experimental task, a multiple branching structure (Fig. 3, middle) is assigned. When order information must be retained, a repeated right-branching structure (Fig. 3, bottom) is assigned. For a scan proceeding from the top nodes of such structures, all of the multiple branches are equally accessible, while each node in a repeatedly right-branching structure can be reached only through the preceding node. Therefore, the recognition latencies produced by any of several scanning strategies should not increase with serial position in the multiple branching case, but should increase with serial position in the right-branching case.

The results of this experiment suggest further research in which the structure of the string is varied independently of serial position. Groupings of the members of the string might be accomplished by introducing hesitation pauses at various serial positions or by selecting the items at different positions from distinct sets of materials (e.g., fruits in first and second position, flowers in third and fourth, etc.) (Bower, 1970).

More subtly, Ss might be made to assign different structure to the same string by varying the task at hand. For example, patterns of RT might reveal that six-digit strings were grouped as three pairs when the task was to recognize two-digit probes, but as two triplets when the task was to recognize three-digit probes. Not only would such groupings demonstrate the independence of structure and serial position per se, but also the different groupings might lead to different subjective mislocations of extraneous sounds occurring during the presentation of the string (Garrett, 1965).

BOWER, $G$ REFERENCES

memory Cognitivanizational factors in 18-46.

GARRETT, M. F. Syntactic structure and judgements of auditory events: A study of the location of extraneous noises in sentences. PhD thesis, University of Illinois, 1965.

STERNBERG, S. Discovery of processing states: Extensions of Donders' method. In W. G. Koster (Ed.), Attention and performance II. Acta Psychologica 30, 1969a, 276-315

STERNBERG, S. Memory scanning: Mental processes revealed by reaction-time experiments. American Scientist, 1969b, 57, 4, 421-457.

(Received for publication June 12, 1972; revision received July 10,1972 , 